\title{
Implementación de un modelo de planeación estratégica para una empresa de servicios de construcción
}

\author{
María Camila Sandoval Acevedo* \\ Mauricio Eduardo Escobar Salas**
}

Recibido: 10 de agosto de 2020

Revisado: 15 de septiembre de 2020

Aprobado: 10 de octubre de 2020

Citar como:

Sandoval Acevedo, M. C. y Escobar Salas, M. E. (2021). Implementación de un modelo de planeación estratégica para una empresa de servicios de construcción. Revista CIFE, 23(38). https://doi.org/10.15332/22484914.6133

\section{Resumen}

Mediante un estudio de caso, el presente artículo tiene como objetivo proponer un modelo de planeación estratégica para determinar las políticas y la metodología para que una empresa de servicios — de carácter familiar, con 14 años de trayectoria, que trabaja en brindar soluciones de mantenimiento y construcción para infraestructura industrial, institucional y residencial - tenga una hoja de ruta clara a seguir, basada en su experiencia y en el modelo de gestión que ha llevado a cabo. Este trabajo responde a la necesidad de dicha empresa de contar con un modelo de planeación estratégica que le permita explorar nuevas metas. Se ha identificado la importancia de pensar a largo plazo, con el fin de potenciar sus fortalezas y aprovechar las oportunidades que ofrece su entorno, así como de reconocer y enfrentar las amenazas en el sector y minimizar las debilidades que se presentan al interior de la organización.

Palabras clave: administración, dirección, estrategia, servicios de construcción, hoja de ruta.

Clasificación JEL: M00, M10, M13, M20.

\section{Implementation of a strategic planning model for a construction services company}

Abstract

Through a case study, the purpose of this article is to propose a strategic planning model to determine the policies and methodology for a services company — a family business

\footnotetext{
*Estudiante MBA Universidad del Rosario. ORCID: https://orcid.org/0000-0002-7213-8303. Correo electrónico: mariaca.sandoval@urosario.edu.co

** Profesor de cátedra Universidad del Rosario. ORCID: https://orcid.org/0000-0002-8900-917X
} 
with 14 years of experience, which provides maintenance and construction solutions for industrial, institutional and residential infrastructure - to have a clear a roadmap to follow, based on its experience and the management model it has carried out. This work corresponds to the need of said company for a strategic planning model that allows it to explore new goals. The importance of long-term thinking has been identified, in order to enhance its strengths and take advantage of the opportunities offered by the environment, as well as to recognize and face the threats of the sector and minimize the weaknesses that arise within the organization.

Keywords: administration, management, strategy, construction services, roadmap.

JEL Classification System: M00, M10, M13, M20.

\section{Introducción}

Esta es una empresa familiar fundada en el año 2006 con la misión de ofrecer soluciones de mantenimiento y construcción en infraestructura industrial, institucional y residencial. Su sede operacional ha sido Pereira, en el departamento de Risaralda, aunque ha desarrollado algunas actividades comerciales en ciudades del Quindío, Caldas, Valle del Cauca y Antioquia.

Por las características del sector de la construcción, especialmente en el caso del mantenimiento de infraestructura, tradicionalmente se ha encargado esta labor a personas independientes, las cuales solían prestar sus servicios informalmente. En su evolución, el sector de la construcción ha sido impulsado y determinado por las regulaciones. Por ejemplo: la Resolución 1409 del 23 de julio de 2012; el reglamento para protección contra caídas en el trabajo en alturas; la separación de los riesgos profesionales, desde la privatización del sistema de seguridad social; y la incorporación del sector financiero como gestor de las empresas de riesgo profesional.

En Colombia, la Red de Cámaras de Comercio (Confecámaras, 2017) afirma que de cada 100 empresas que fueron creadas en 2012, solo 34 siguen en funcionamiento después de 5 años. El fracaso de los emprendimientos se explica, de acuerdo con Pardo (2015), por tres factores principales: financiero (falta de financiación o mal manejo financiero), administrativo (carencia de planeación y de indicadores de gestión, principalmente), y mercadeo (desbalance entre precio, producto, promoción y plaza).

Las empresas que logran mantenerse en el mercado tienen un reto adicional: aumentar la productividad. A diferencia de la competitividad, la productividad - como lo menciona el Consejo Privado de Competitividad (CPC, 2018) - consiste en generar valor agregado por la vía de mayor eficiencia en la producción. En el informe de productividad de la Cámara Colombiana de la Construcción (Camacol, 2018), se indica que la productividad de una empresa depende en un $65 \%$ del manejo de sus factores internos, como la ganancia en sus procesos internos y la demanda de productos; y en un $35 \%$ en los factores externos. 
Dentro de este contexto, y motivado por las fuerzas del entorno, se considera fundamental analizar las prácticas que le han permitido a la empresa permanecer en el mercado, identificar las oportunidades o amenazas externas y conocer los problemas internos que puedan estar impidiendo su crecimiento o pongan en peligro su sostenibilidad. Los socios se han planteado, como objetivo a corto plazo, promover la sostenibilidad y el crecimiento de la organización basado en la expansión de la operación a otras regiones.

\section{Historia}

Esta empresa fue fundada por Gloria y Sergio, una pareja de esposos ingenieros civiles nacidos a principios de la década de los sesenta, cada uno con más de 30 años de experiencia profesional. Ellos han desarrollado proyectos principalmente en construcción, mantenimiento y diseño de vías y edificaciones, con lo que han logrado ser reconocidos por su trayectoria.

Después de hacer múltiples intentos por desarrollar negocios de ingeniería, tocar puertas en los sectores público y privado, trabajar para algunas empresas y asociarse con otras personas, decidieron, en 2006, fundar esta empresa. Desde sus experiencias de éxitos y fracasos, consideraron que, entre los dos, podían crear una empresa de ingeniería innovadora, generar empleos y tener una visión a largo plazo.

Gloria dedicó la mayor parte de su vida profesional a contratar con el Estado, pues es este el que normalmente genera la mayor inversión en infraestructura. Sin embargo, durante la primera década del 2000, la participación en la contratación estatal se comenzó a limitar, porque, aunque regida por la Ley 80 de 1993, los procesos se hacían muy vulnerables a la corrupción, les generaba a los proponentes mucho desgaste administrativo y alto costo de oportunidad. El hecho de que el acceso a los contratos públicos fuera cada vez más improbable, se convirtió en el factor que generó el nacimiento de una empresa que pudiera llegar a otros mercados.

Sergio ha trabajado para importantes empresas del sector de la construcción en el país, participando en proyectos destacados. En el año 2006 tuvo la oportunidad de trabajar dentro de un proyecto en Panamá y, en este nuevo contexto, junto con su esposa, percibieron que muchas personas que trabajaban en la construcción no concentraban su actividad comercial en la contratación estatal, sino que se enfocaban en prestar servicios para el sector privado, tener sus propios proyectos y generar un portafolio dirigido a satisfacer este mercado, el cual había sido casi inexplorado hasta ese momento por ellos.

\section{Acceso a recursos financieros}

En 2006, luego de volver a Colombia, vieron la primera posibilidad de negocio enfocada en el sector privado: un proyecto inmobiliario de subdivisión de vivienda sobre una casa con una muy buena ubicación que fue considerada en su momento una oportunidad. 
Jardín de Álamos es un proyecto inmobiliario compuesto por 8 apartamentos, algunos fueron vendidos en cierto porcentaje y otros se mantienen en propiedad de los socios. Este proyecto se logró finalizar exitosamente con créditos obtenidos de entidades bancarias. Contrario a lo que se podría pensar, cumplir con las obligaciones financieras no fue un obstáculo; se convirtió en una de las mayores motivaciones para continuar, "teníamos que conseguir más proyectos, buscar negocios, hablar con amigos", dice su socia y gerente. Así la empresa se vio obligada a continuar su dinámica y comenzó a trabajar en otras actividades como el mantenimiento y construcción de infraestructura para la industria.

\section{Fuente interna de recursos}

Desde el principio, Gloria asumió la gerencia de la compañía, mientras que Sergio actuó como un asesor externo para la empresa y continuó trabajando para otras. Ambos consideran que esto fue fundamental para darle solidez en los primeros años, pues la familia no dependía económicamente de su propia empresa, lo que les permitía reinvertir las utilidades que se pudieran generar en capitalizar y desarrollar otros negocios.

Durante el tiempo de existencia de la empresa se han superado múltiples pruebas. Recogiendo las palabras de la gerencia, se han probado y descartado ideas, pero la mayor parte del tiempo ha transcurrido solucionando las contingencias del día a día. A pesar de esto, una de las ideas más importantes que ha surgido en la búsqueda de nuevos negocios para dar sostenibilidad a la actividad, para aprovechar la capacidad instalada y el conocimiento del sector, fue la comercialización de productos de construcción. A pesar de esto, en 2018, en la búsqueda de nuevas opciones de negocio para dar sostenibilidad a la actividad, se diseñó una propuesta de comercialización de productos de construcción, una ferretería propia, llamada 'Su Mano Derecha'. Para concretarla, se conectaron las fortalezas de la capacidad instalada, el conocimiento del sector, contar con un local propio justo al lado de la sede administrativa de la empresa y saber, de primera mano, las necesidades en el campo de la construcción.

\section{Nuevo escenario}

En el año 2019, los socios, apoyados en su nueva socia, su única hija, decidieron documentar las prácticas administrativas desarrolladas en la empresa y generar un modelo de planeación que les permitiera expandir su operación. Esto, consolidando un marco de políticas que permitiese mantener la esencia y filosofía de la empresa, que ha sido el sello de confianza y calidad en el que han basado su reputación. 


\section{Concepto de planeación estratégica}

Figura 1. Escenario para la planeación estratégica

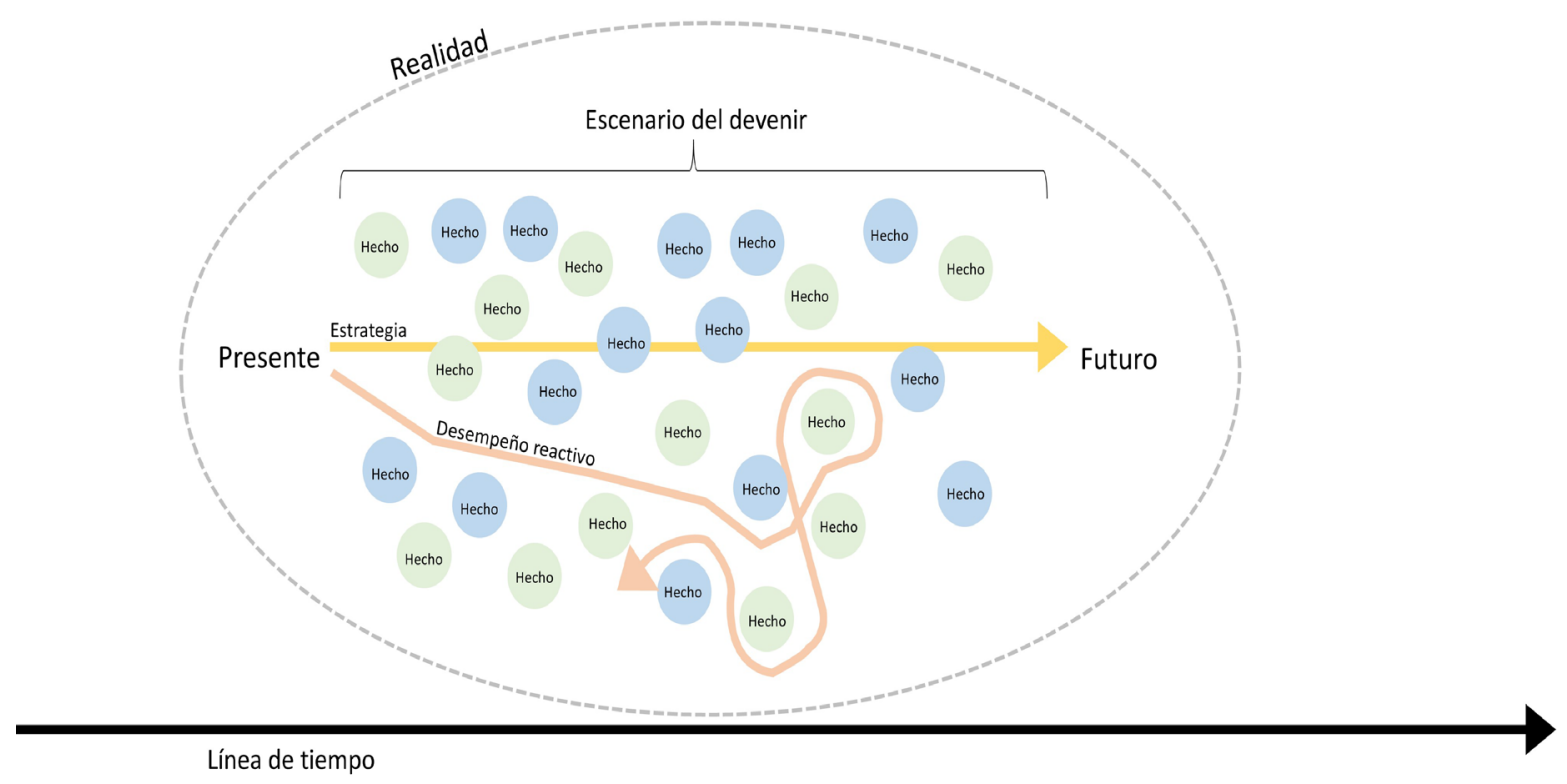

La realidad empresarial se mueve con el paso del tiempo y está compuesta por el escenario del presente, del devenir y del futuro. En la figura 1 se ejemplifica cómo desde el presente se está construyendo el futuro deseado, pero entre estos dos puntos existe un trayecto lleno de incertidumbres, hechos incontrolables y elementos emergentes, que exigen la claridad de ese elemento trascendente, ubicado más allá del devenir para evitar una posición pasiva. Contar con una herramienta para afrontar el escenario del devenir es un factor clave con el cual las organizaciones previenen un desempeño reactivo, al quedarse abrumadas en la emergencia de hechos, el desperdicio de recursos y, finalmente, perder la claridad de sus objetivos.

Dada la complejidad del presente, se necesitan instrumentos para, por una parte, interpretar la realidad y, por otra, gestionar la información que otorgue sentido a todos los sucesos que se reconocen en el contexto. El riesgo es no alcanzar a percibir los hechos y tomarlos como acontecimientos aislados, quedando la empresa sin una lectura clara de su realidad. A partir de esta situación, han surgido posturas y modelos que brindan esos instrumentos a la empresa para diseñar una hoja de ruta que minimice la incertidumbre, así, esta hoja de ruta será su estrategia.

Normalmente la interpretación de los hechos es subjetiva y termina dependiendo de la mirada del que ordena, analiza e interpreta lo que percibe. El concepto de planeación estratégica que, 
según Pulido (2009), es un desarrollo formal que asegura captar la información relevante para tomar decisiones, también establece como protagonistas a los ejecutantes, pues es según el criterio de los miembros de la organización que se diseña, implementa y gestiona la hoja de ruta. Este proceso, de acuerdo con Manucci (2010), es dinámico e implica la toma de decisiones, pues se debe asumir el riesgo de proyectar un futuro sobre un terreno de hipótesis.

Toda organización hace parte de un entorno en movimiento, que se construye a partir de fotografías de momentos. La utilidad de estas depende de la calidad y cantidad de información veraz con la que se construyan, ya que, al final, resultarán en una especie de película que narrará lo ocurrido durante un tiempo. De este modo surge un reto para todas las organizaciones: mantener una mirada constante y capturar las mejores imágenes con la velocidad necesaria, para procesar rápidamente los hechos, y ajustar y actuar en el momento indicado. Algunas características relevantes sobre un modelo de planeación estratégica es que llegue a ser flexible, dinámico y lógico.

En Pulido (2009), los modelos se trabajan sobre dos etapas: en la primera se analizan las características internas y externas, se determinan los objetivos y se elige una estrategia; en la segunda, se ejecutan las acciones y se hace seguimiento. Adicionalmente, Pulido presenta algunas teorías, como la expuesta por Michael Porter, quien considera que la planeación no se puede desvincular de la implementación; la de Ohmae, que establece que este debe ser un proceso natural y racional; o la de Mintzberg, quien enfatiza en la realización de un proceso espontáneo que involucra a todos los niveles de la organización y no solo a la alta gerencia.

Otra propuesta es la de Manucci (2006), que contempla un proceso básico de tres etapas: la primera, en la que se define la realidad corporativa que involucra las creencias, emociones y experiencias; la segunda, que contempla la hipótesis de intervención para llegar al futuro deseado; y, por último, la gestión de resultados. Algo importante para resaltar de este autor es lo valioso que resulta, dentro de su teoría, mantenerse como partícipe en el contexto dinámico y tomar posición, pues de ese modo se desarrollan habilidades en el pensamiento para responder rápidamente con estrategias.

\section{Aplicación de un modelo de planeación estratégica}

La elección del modelo de planeación estratégica a implementar depende del estratega y lo que este considere más útil para su fin, de acuerdo con su propia realidad. Para desarrollar el modelo de planeación estratégica para esta empresa, la herramienta a utilizar será el propuesto por David (2011). De acuerdo con este autor, "las empresas que usan conceptos de planeación estratégica muestran importantes mejoras en ventas, rentabilidad y productividad con respecto a las empresas que no cuentan con un sistema de planeación de actividades" (David, 2011, p. 17). 
El modelo tiene varias características que resultan atractivas para implementarlo dentro de esta empresa. Incluye el proceso integral que mencionaba Pulido (2009), contando con una etapa de pensamiento estratégico en la que se dan cita una mirada interna y otra externa, lo que sirve para para determinar la posición y permite, también, una evaluación y priorización de estrategias. Posteriormente, se proponen herramientas para la acción y su seguimiento, lo que le hace un instrumento cíclico, como se ilustra en la figura 2, que permite realizar una lectura permanente de la organización y de la forma en la que está invirtiendo sus recursos, siempre en función de los objetivos planteados.

Figura 2. Ciclo planeación estratégica

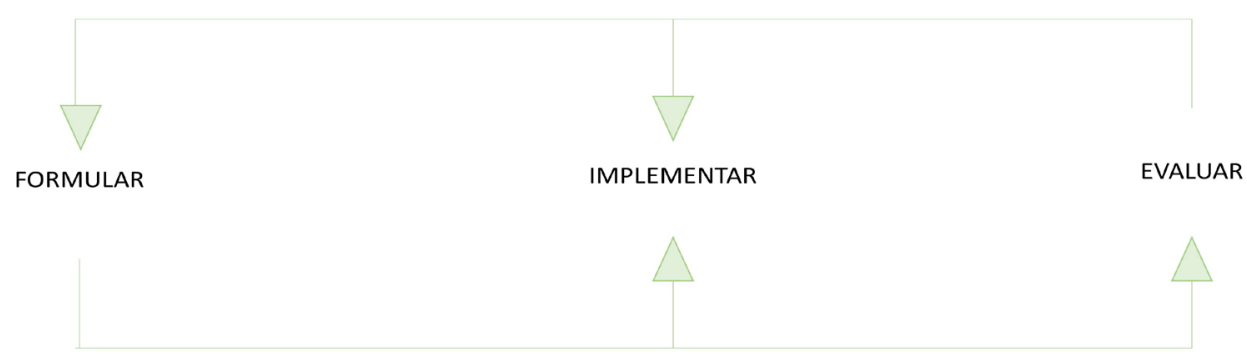

Esta metodología incluye visiones de otros autores mencionados antes, como Porter, utilizando su metodología para diagnosticar la posición de la organización en su entorno; además, contempla otros elementos, mencionados por Manucci (2006), que sirven para evaluar internamente las creencias, emociones y experiencias, teniendo en cuenta la intuición desarrollada por los integrantes de la organización.

\section{Metodología}

Para el desarrollo de este estudio de caso se usó el modelo integral del proceso de planeación estratégica de David (2011), que se presenta en la figura 3. 
Figura 3. Modelo integral de gestión estratégica (Comprenhensive Model of the Strategic Management)

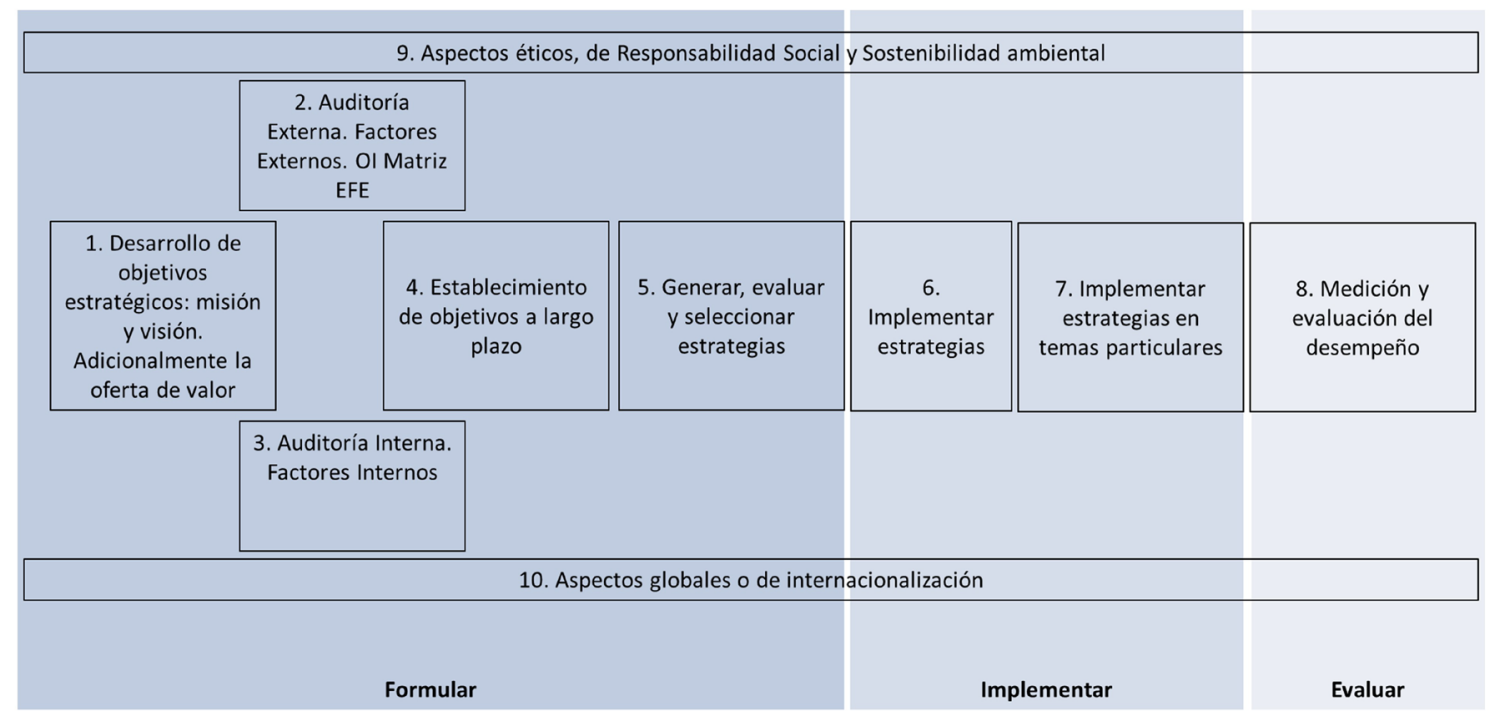

Fuente: David (2011, p. 94).

La implementación de este modelo implica, en primer lugar, revisar el programa de gestión con en el que cuenta la organización y realizar una evaluación de los factores externos utilizando el modelo de las fuerzas competitivas de Porter (David, 2011), determinando así el nivel de impacto de cada una sobre la empresa. Posteriormente, se evalúan los factores internos: estructura interna de la organización y cultura organizacional, estrategia de marketing, gestión financiera, gestión de operaciones, investigación y desarrollo, manejo y administración de la información. Con estos datos se construye la matriz de evaluación de factores internos y externos, para determinar cuantitativamente la incidencia de los fenómenos identificados y así contar con un diagnóstico completo de la organización. Con este instrumento, y mediante el uso de la matriz DOFA, se establecen estrategias con base en los elementos positivos y negativos del diagnóstico, para proponer, finalmente, un plan para la implementación de estas.

Para llevar a efecto el anterior proceso en el estudio de caso, se utilizaron como insumos: la revisión de fuentes bibliográficas para la comprensión del entorno del sector; entrevistas a miembros de la empresa con guiones preestablecidos, con el fin de obtener la mayor información necesaria para conocer la situación interna y la percepción desde diferentes puntos de vista. También se recolectó y analizó la información financiera y del plan de gestión. Finalmente, se realizaron visitas a la sede principal, las obras en ejecución y la ferretería. 


\section{Plan y recomendaciones de implementación y aplicación}

Las sugerencias que se dan a la empresa para el cumplimiento de los objetivos planteados, son el resultado de implementar las herramientas que propone el modelo de planeación estratégica de David (2011): definir la misión y visión; luego, evaluar los factores internos y externos, y, posteriormente, construir la matriz DOFA (debilidades, oportunidades, fortalezas y amenazas). Finalmente, se utiliza la matriz cuantitativa de planeación estratégica, Quantitative Strategic Planning Matrix (QSPM), para así tener como resultado la priorización de las 15 estrategias resultantes de la matriz DOFA. Los resultados de la aplicación de la matriz se presentan en orden de prioridad en la tabla 1.

Tabla 1. Resultado matriz QSPM

\begin{tabular}{|c|c|c|}
\hline N. ${ }^{\circ}$ & Resultados estrategias - DOFA & Puntaje \\
\hline 1 & $\begin{array}{l}\text { Crear objetivos a largo, mediano y corto plazo para aumentar los } \\
\text { factores de éxito. }\end{array}$ & 4,32 \\
\hline 2 & $\begin{array}{l}\text { Crear un plan estratégico que permita despersonalizar la empresa de } \\
\text { la figura de la gerente. }\end{array}$ & 3,75 \\
\hline 3 & Tener operaciones permanentes de la empresa en otras ciudades. & 3,43 \\
\hline 4 & $\begin{array}{l}\text { Implementar una estrategia de mercadeo, para que llegue a nuevos } \\
\text { clientes la promesa de valor que tiene la empresa y diversificar los } \\
\text { clientes actuales. }\end{array}$ & 3,19 \\
\hline 5 & $\begin{array}{l}\text { Tener como política que el lugar donde opere la empresa tenga una } \\
\text { ferretería anexa de propiedad de la empresa. }\end{array}$ & 3,11 \\
\hline 6 & Crear un proceso para la gestión de los recursos humanos. & 2,49 \\
\hline 7 & $\begin{array}{l}\text { Contratar una consultoría para tener información sobre los } \\
\text { competidores y poder medirse con el mercado. }\end{array}$ & 2,39 \\
\hline 8 & $\begin{array}{l}\text { Diseñar un organigrama de la organización con el que se puedan } \\
\text { cumplir los objetivos propuestos, que incluyan perfiles de cargos y } \\
\text { tareas concretas. }\end{array}$ & 2,34 \\
\hline 9 & $\begin{array}{l}\text { Contratar por proyectos empresas especializadas en mano de obra } \\
\text { que cumplan con los requisitos de seguridad industrial y laborales. }\end{array}$ & 2,24 \\
\hline 10 & $\begin{array}{l}\text { Crear indicadores para medir el comportamiento de la empresa en } \\
\text { cada línea de negocio, para medir el avance hacia los objetivos. }\end{array}$ & 2,1 \\
\hline 11 & Licitar en el sector público y diversificar los clientes. & 1,98 \\
\hline 12 & $\begin{array}{l}\text { Establecer un indicador para medir las inversiones que se realizan, } \\
\text { bajo la filosofía de la empresa de contar con escenarios } \\
\text { conservadores. }\end{array}$ & 1,75 \\
\hline
\end{tabular}




\begin{tabular}{llc}
\hline N. & Resultados estrategias - DOFA & Puntaje \\
\hline 13 & $\begin{array}{l}\text { Contar con parámetros para la negociación con proveedores de } \\
\text { materiales de construcción utilizados como insumos para la ferretería } \\
\text { y la ejecución de obras. }\end{array}$ & 1,56 \\
\hline 14 & $\begin{array}{l}\text { Crear un programa para la implementación de tecnologías en los } \\
\text { diferentes procesos de la empresa: mercadeo, administrativos, } \\
\text { operativos, gestión de la información. }\end{array}$ & 1,54 \\
\hline 15 & Implementar un modelo de gestión de información de los proyectos. & 0,89 \\
\hline
\end{tabular}

Para la ejecución de las estrategias resultantes, se recomienda el plan que se muestra en la figura 4, el cual se distribuye en dos fases: planeación de la estrategia corporativa y la implementación de los objetivos estratégicos.

Figura 4. Cronograma implementación estrategias FASE I y FASE II

\begin{tabular}{|c|c|c|c|c|c|c|c|c|c|c|c|}
\hline MES 1 & MES 2 & MES 3 & MES 4 & MES 5 & MES 6 & MES 7 & MES 8 & MES 9 & MES 10 & MES 11 & MES 12 \\
\hline \multicolumn{4}{|c|}{ FASE I } & \multicolumn{8}{|c|}{ FASE II } \\
\hline \multicolumn{4}{|c|}{ ESTRATEGIA CORPORATIVA } & \multicolumn{8}{|c|}{ IMPLEMENTACIÓN OBJETIVOS ESTRATÉGICOS } \\
\hline
\end{tabular}

Las fases se enfocan en cumplir unas estrategias que para su ejecución se desglosan en acciones. Por esta razón, se incluyen dentro del plan de acción: tareas, recursos, tiempo de inicio, periodicidad de ejecución y responsable dentro de la organización. Durante las fases es importante implementar herramientas de seguimiento y control que permitan verificar el desarrollo de las tareas para lograr las metas. Por tal razón, también se recomienda el seguimiento a los indicadores.

La FASE I comprende el desarrollo de las estrategias No. 1 y No. 2, que se refieren a metas relacionadas con la estrategia corporativa. La FASE II contiene el desarrollo de las estrategias No. 3 a No. 15, que se refieren a metas que incluyen el logro de objetivos estratégicos, los cuales se deben revisar y ajustar si es el caso. Como su implementación depende de los resultados de la FASE I, no se especifican sus acciones dentro de este documento.

El desarrollo de las estrategias está en manos de diferentes niveles de la organización, como se representa en la figura 5. La FASE I tiene como responsables a los accionistas y a la junta directiva, mientras que la ejecución de la FASE II corresponde a la gerencia, apoyada en el nivel operativo. 
Figura 5. Ejecución del plan de acuerdo con los niveles de la organización
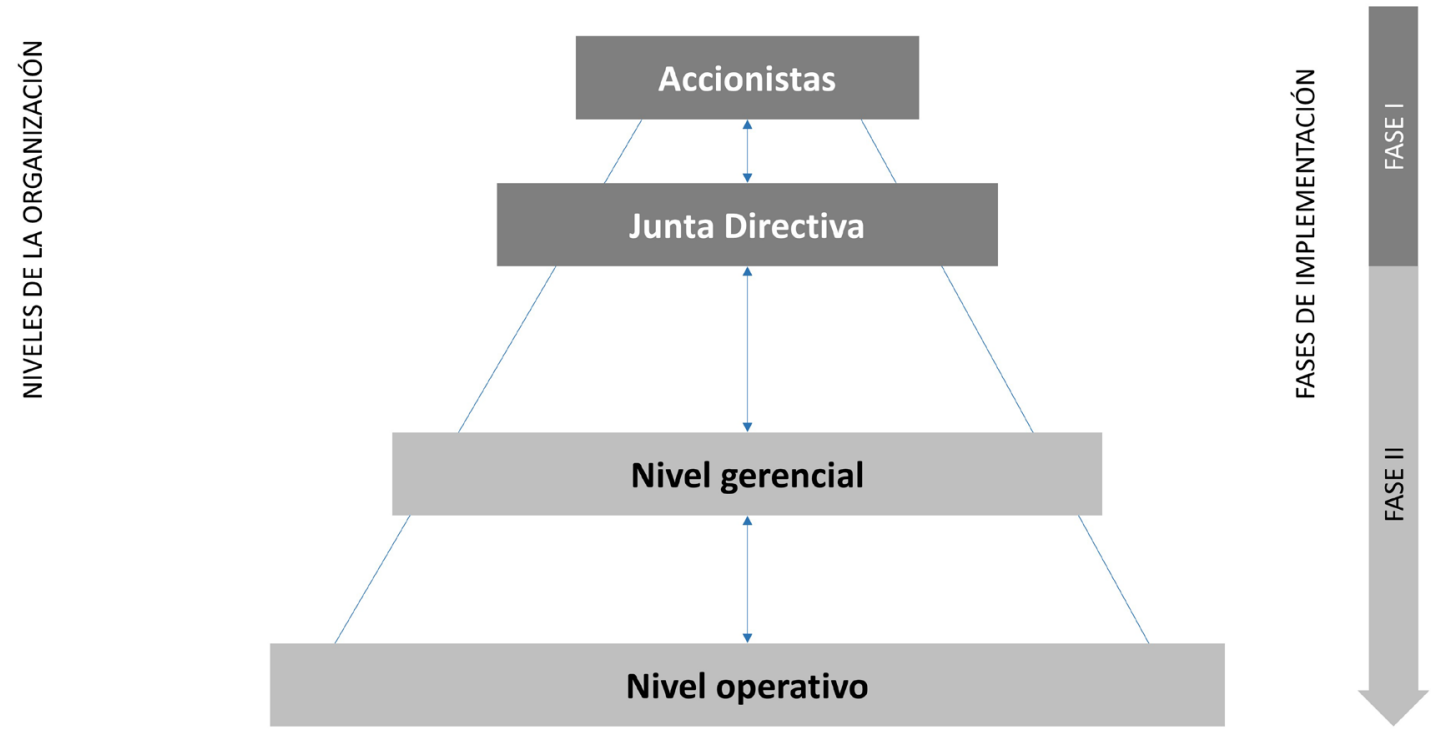

\section{FASE I: estrategia corporativa}

De acuerdo con el diagnóstico de la situación a 2019, se recomienda el desarrollo de 2 estrategias iniciales enfocadas en un ejercicio interno para reflexionar sobre el propósito de la organización, a qué se dedica, para quiénes y cuál es la visión a futuro. La empresa lleva toda su trayectoria con la preocupación de mantener su sostenibilidad y no se ha tomado un tiempo para revisar su propósito y los escenarios futuros que le permitan plantearse nuevos objetivos estratégicos. Es por eso que se recomienda, como primer paso, implementar acciones que le permitan sentar las bases para la toma de decisiones y el manejo de los recursos dentro de un periodo de tiempo determinado, previendo una posible transición generacional en la gerencia.

Para la implementación de las estrategias, se plantea un esquema en niveles de ejecución dividido en: estrategia, acciones y tareas, como se muestra en la figura 6. En cada nivel se asignan recursos, fecha de inicio, periodicidad, responsable y resultado esperado; pues es vital tener una herramienta clara para verificar el cumplimiento de cada paso y garantizar llegar a la meta esperada. 
Figura 6. Esquema estructura implementación estrategias

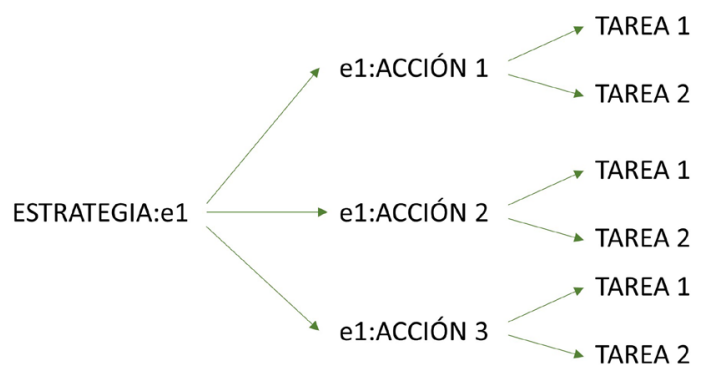

Para el desarrollo de esta fase se definen las acciones y tareas en las que se detallan, para cada uno de los niveles anteriores, los recursos, responsables, tiempos e indicadores correspondientes. A continuación, se describen las dos estrategias para llevar a cabo esta fase:

- Revisar y formular objetivos a corto, mediano y largo plazo de acuerdo con la visión estratégica. La meta es contar con la misión concreta y una visión ambiciosa pero realista para formular los objetivos estratégicos de la organización que permitan cumplir con la visión.

- Crear un plan estratégico que permita despersonalizar la empresa de una persona clave: la gerente. La meta es que la empresa cuente con una matriz de mando para la ejecución de los objetivos estratégicos.

El seguimiento a las tareas propuestas y la verificación de su cumplimiento durante esta etapa se encuentra liderada por la gerencia. La ejecución depende de un trabajo en conjunto con la junta directiva que, para el caso, son los mismos accionistas. Es importante garantizar la creación de estos espacios y que los participantes lleguen con una visión amplia del negocio que enriquezca las discusiones.

\section{FASE II: implementación de objetivos estratégicos}

Una vez implementada la FASE I, se deben revisar los resultados obtenidos y, de acuerdo con estos, validar las 13 estrategias restantes. En esta etapa se plantea la ejecución de los objetivos estratégicos mediante un plan de acción realista para alcanzarlos. De acuerdo con la planeación propuesta, el plan de acción iniciaría con los objetivos a corto plazo que tienen una vigencia de un año.

La ejecución de la FASE II está en cabeza de la gerencia y su equipo de trabajo, por lo cual, deben reunirse periódicamente con su equipo, para revisar en forma cooperativa los asuntos operativos y el progreso en relación con los planes, para identificar los riesgos y tomar decisiones. 
El reto durante este tiempo está en el seguimiento y monitoreo de los procesos clave, para generar alarmas oportunas que permitan hacer los ajustes correspondientes con el fin de lograr los objetivos propuestos y, si es el caso, hacer los ajustes a los objetivos proyectados. Los resultados de esta ejecución deben comunicarse periódicamente a la junta directiva. También hay que tener presente que el trabajo de la junta directiva no termina cuando la FASE I propuesta llegue a su fin.

\section{Conclusiones}

Una empresa de servicios de construcción como la estudiada a lo largo del documento, representa solo una muestra de las pequeñas empresas familiares que componen el ecosistema empresarial colombiano, y es un ejemplo de cómo se puede desarrollar un emprendimiento dentro del contexto del país, que genera empleos fijos con garantías legales, beneficiando a familias al contar con empleo formal y permanente. Evidencia, además, cómo algunas de estas empresas nacen y logran sostenerse en su primera generación, gracias al liderazgo carismático de sus dueños y gerentes, pero tienen dificultades para ceder la dirección a la segunda generación. Esto, debido a que no tienen sentadas ni definidas las bases estructurales de la organización ni sus objetivos a corto, mediano y largo plazo por medio de una planeación estratégica. En este caso, la empresa ha logrado sostenerse en sus primeros años, pero afronta el reto de sobrevivir a la transición generacional y mantener su éxito comercial, sin depender de las personas que la crearon.

De acuerdo con lo anterior, se obtuvieron una serie de conclusiones que se agrupan de la siguiente forma:

1. Buenas prácticas tomadas de la trayectoria que pueden servir como sugerencias para el entorno empresarial, con relación a 4 temas: 1) desarrollo de un modelo de negocio y generación de valor; 2) gestión financiera; 3) cultura organizacional; y 4) adaptabilidad ante factores externos.

2. Herramientas para el fortalecimiento de una organización con características similares: pyme familiar en el sector de la construcción.

Las buenas prácticas comienzan con determinar el modelo de negocio y dónde se crea valor, proceso que requirió madurez en la visión de los socios. Esta empresa tiene la misión de prestar servicios de mantenimiento y adecuaciones a infraestructura para que los propietarios conserven el valor de sus propiedades en el tiempo. Esta actividad implica atender necesidades a diario de diferente magnitud y complejidad, que exigen contar con una capacidad de respuesta inmediata por parte de la empresa, en el tiempo justo y con calidad garantizada. 
En cuanto a la gestión financiera, el manejo de los recursos se puede denominar conservador. La empresa ha logrado sobrevivir a sus casi quince años de existencia a pesar de presentar altibajos en las ventas, sosteniendo dos principios: no mezclar las finanzas familiares con las empresariales, y mantener la liquidez. Esto significa que: 1) no se corren riesgos que impliquen disminuir el capital de trabajo; 2) se reinvierten las utilidades; y 3) no se recurre a fuentes de financiación que pongan en peligro el patrimonio.

En experiencias anteriores, los socios intentaron crear negocios cuando alguno de los dos había perdido su empleo, para así reemplazar sus ingresos familiares, pero no fueron rentables. Por eso, cuando emprendieron esta empresa, concluyeron que requerían contar con una mínima planeación administrativa, financiera y de mercadeo con visión a largo plazo, dada la dificultad para que de manera inmediata se pudieran obtener ingresos suficientes para su operación, cumplir con los costos financieros y obtener un retorno de la inversión.

Conocer el modelo de negocio, cuáles son sus clientes y dónde se genera valor ha generado un modelo intuitivo que sirve a la gerencia para determinar los negocios que son atractivos para la empresa, teniendo en cuenta el nivel de riesgo que se ha manejado. Lo anterior, una vez la organización ha entendido que una empresa logra consolidarse con el tiempo, apoyada en el convencimiento de que se trata de una carrera de largo aliento, por lo que resulta conveniente dosificar el esfuerzo para no sufrir un colapso prematuro. Se sugiere, entonces, que el interesado en crear su propio negocio planee con antelación su idea con decisión de trabajo a largo plazo.

Otra de las prácticas financieras de la empresa es la forma en que se han utilizado las ganancias generadas, pues estos recursos se reinvierten en la empresa y permiten autofinanciar obras y diversificar negocios, lo que se ha convertido en uno de los principales factores para fortalecer su competitividad. Se recomienda al nuevo empresario que cuente con diversidad de actividades para obtener el ingreso de su grupo familiar, ya que esto le permite a la organización contar con el capital requerido para que sea sostenible en el tiempo.

En cuanto a la cultura organizacional, la empresa ha adoptado los principios de trabajo de la gerente, estos son: confianza, responsabilidad, austeridad, creatividad y persistencia. El reto está en lograr transmitir el mensaje a todos los integrantes de la organización, especialmente por la dinámica propia del gremio de la construcción.

A diferencia de la mayoría de empresas de servicios del sector, la empresa cuenta con empleados de construcción fijos. Esto, como se indicó en el estudio, puede ser un aspecto que genere vulnerabilidad financiera en caso de no lograr las ventas esperadas, sin embargo, es una garantía para mantener la filosofía de la empresa y ofrecer servicios con un estándar de calidad alto, lo que se traduce en credibilidad y confianza por parte de los clientes. 
La capacidad de adaptación que tenga la organización ante los factores externos es fundamental para su supervivencia, pues cada empresa hace parte de un ecosistema dinámico y resulta clave tener una lectura constante de lo que está pasando en el entorno. No obstante, hasta el momento y a pesar de su afán por mantenerse productiva, esta empresa no ha dedicado muchos esfuerzos a la evaluación de este tema.

Si bien es cierto que, en la actualidad, y ante la aparición de nuevas tecnologías, se generan emprendimientos que rápidamente alcanzan altos niveles de rendimiento económico $\mathrm{y}$ productividad, también resultan ser casos especiales. Lo más probable es que iniciativas que prestan servicios tradicionales, como la construcción, confección, alimentación o agroindustria, busquen herramientas que les permitan ir a la par de las necesidades de los clientes, para ser más competitivos.

Pero también hay factores que hacen parte de intervenciones estatales, lo que evidencia la vulnerabilidad de la pequeña empresa ante las crisis. Los requerimientos de seguridad y salud en el trabajo para el sector de la construcción son muy altos, así que para una pyme con pocos empleados su cumplimiento se hace muy oneroso. Por ejemplo, se debe contar con un profesional de seguridad y salud en el trabajo así se tenga solo un empleado de construcción. Se recomienda, entonces, conocer la regulación estatal pues, sin duda, influye en los comportamientos financieros de las empresas.

En cuanto a las herramientas para el fortalecimiento de una organización, se concluye que, previo a su utilización, se requiere contar con conocimiento suficiente acerca de la empresa, para determinar el momento que atraviesa y a qué retos se está enfrentando. En este caso, la empresa ha mostrado un comportamiento financiero sostenido, en el que aumenta los niveles de liquidez año a año, por eso la prioridad al momento de realizar este análisis está en el fortalecimiento de las bases estratégicas, lo que posteriormente se traducirá en acciones.

La evidencia consultada reconoce la importancia de que las pymes cuenten con herramientas de administración para su supervivencia y sostenibilidad. Por esto, resulta fundamental para esta empresa implementar un modelo de planeación estratégica, que le permita establecer objetivos acertados y alcanzar nuevas metas. Se ha identificado la prioridad de pensar a largo plazo, pero actuar en el corto, potenciando sus fortalezas y aprovechando las oportunidades que ofrece el entorno. Dicha estrategia contemplará también reconocer y actuar ante las amenazas en el sector, así como minimizar las debilidades que se presentan al interior de la organización.

Adicionalmente, contar con un plan estratégico para la administración brinda el soporte necesario para la consolidación de la misión y visión. Este plan prevé involucrar a los niveles operativos y gerenciales como un recurso valioso de la organización, que aporta innovaciones en los procesos y busca soluciones de acuerdo con las necesidades, utilizando las últimas tecnologías del sector para ofrecer un servicio de calidad. 
A lo largo del escrito se establece la importancia de que toda pyme estructure una organización para su consolidación a largo plazo. Sin embargo, dentro de esta experiencia se concluye que, previo a conformar una compleja estructura organizacional, en las fases tempranas del desarrollo de una empresa se hace fundamental la presencia y liderazgo de una persona clave. Este es el motor para mantener el foco y la fortaleza que le garantizan a la empresa su permanencia en el mercado durante su formación inicial. Una vez superada esta etapa, llega el momento de pensar en la arquitectura empresarial, para delegar la toma de decisiones y la supervisión de las tareas.

Para verificar el cumplimiento de los objetivos planteados, es necesario contar con un sistema sencillo de seguimiento y control de ejecución del plan estratégico, por eso se propone un plan de acción que contemple indicadores. Este modelo se puede usar para implementar las estrategias y acciones que se planteen a futuro como punto de partida para medir los avances en el trabajo realizado.

También se resalta la importancia que tiene la implementación de prácticas de gobierno corporativo en una pyme familiar, pues constituyen el medio para alcanzar nuevos retos y para contar con una estructura interna que permita generar confianza con los grupos de interés. Adicionalmente, permiten hacer la transición a las siguientes generaciones, dando herramientas para delegar, controlar y tomar decisiones en colectivo, garantizar transparencia en la información, medir el seguimiento de objetivos y disminuir el conflicto de interés entre gerencia y junta directiva. Se resalta que, como condición para iniciar el cambio hacia estos modelos, dentro de la empresa se debe tener conciencia de la necesidad de implementar esta estructura, sobre todo en esa persona clave que ha sido la cabeza de la organización desde que fue establecida.

Por último, en esta empresa, cada emprendedor es consciente de sus capacidades y talentos. Por ello, es importante: 1) identificar qué líder emprendedor tiene realmente una visión a largo plazo de la empresa y reconoce su capacidad personal para lograr los objetivos planteados por esta; 2) explorar la fuerza vital del emprendedor; y 3) determinar si con base en esa fuerza o fuente de energía, ese líder logra soportar el duro trabajo que requiere conformar una empresa. No se puede pretender que todo individuo se convierta forzosamente en emprendedor, pues esto solo puede ser fuente de frustraciones.

Dada la importancia que tiene para la economía colombiana fortalecer la creación de microempresas que tengan un soporte administrativo y legal totalmente integrado en la economía, y que, por consiguiente, sean sostenibles y perduren en el tiempo, los resultados del presente trabajo trascienden los intereses de la empresa estudiada y constituyen una invitación a poner los ojos sobre este tipo de iniciativas, en especial el sector público, que podría convertirse en el principal aliado de las pymes, para el beneficio de la sociedad y la economía del país. 


\section{Referencias}

ANIF. (febrero de 2014). Las Pymes de Ingeniería y su papel en el sector transporte. Obtenido de http://www.anif.co/sites/default/files/investigaciones/anifpymedeingenieria-0214.pdf

Banco Interamericano de Desarrollo. (2018). Informe macroeconómico de Ámerica Latina y el Caribe 2018: la hora del crecimiento. Obtenido de https://publications.iadb.org/publications/english/document/2018-Latin-Americanand-Caribbean-Macroeconomic-Report-A-Mandate-to-Grow.pdf

Cámara Colombiana de la Construcción - CAMACOL . (2019). Manual de gobierno corporativo para PYMES del sector de la infraestructura. Bogotá.

Cámara Colombiana de la Construcción - CAMACOL. (2018). Informe de Productividad sector construcción de edificaciones. Bogotá.

Consejo Privado de Competitividad - CPC. (2018). Informe Nacional de Competitividad 2018-2019. Bogotá.

DANE. (2019). Boletín técnico Indicadores Económicos Alrededor de la Construcción: III Trimestre 2019. Bogotá.

DANE. (2019). Boletín Técnico Producto Interno Bruto. III Trimestre 2019.

DANE. (2019). Boletín Técnico: Principales indicadores del mercado laboral. Septiembre 2019. Bogotá.

David, F. R. (2011). Strategic Management concepts and cases. Pretince Hall Pearson.

Manucci, M. (2006). La estrategia de los cuatro círculos . Bogotá: Grupo Editorial Norma.

Manucci, M. (mayo de 2010). Mapas para la complejidad: liderazgo, incertidumbre y estrategia. Revista Universidad y Empresa, 8-19.

Pardo, C. (2015). Análisis de los principales factores del fracaso de los emprendimientos en Colombia. Failure Institute.

Pulido, M. B. (2009). Estrategia y modelos estratégicos: aproximación desde la teoría. Bogotá: Universidad Externado de Colombia.

Red de Cámaras de Comercio CONFECAMARAS. (2017). Determinantes de la supervivencia empresarial en Colombia. 


\section{ĆIF́̈ 38}

Umaña, Y. (2003). El sector de la construcción: un sector líder. Obtenido de https://www.google.com/url? sa=t\&rct=j\&q=\&esrc=s\&source=web\&cd=1\&ved=2a hUKEwjXx8jU0 LlAhUwx1kKHbTlARgQFjAAegQIAxAC\&url=https $\% 3 \mathrm{~A} \% 2 \mathrm{~F} \%$ 2Fwww.superfinanciera.gov.co $\% 2$ Fdescargas $\% 3$ Fcom $\% 3$ Dinstitucional $\% 26$ name $\%$ 3DpubFile1000802\%26downloadname\%3DYolimaUma\%25F1a.pdf\&u

$$
\text { (c) (i) (?) }
$$

